\title{
Characterization of organic ultra-thin film adhesion on flexible substrate using scratch test technique
}

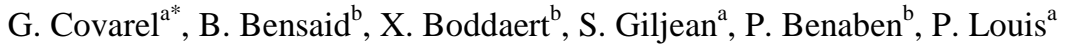 \\ ${ }^{a}$ Laboratoire de Physique et Mécanique Textiles EA CNRS 7189, Université de Haute Alsace, 61 rue Albert \\ Camus 68093 Mulhouse Cedex. France \\ ${ }^{\mathrm{b}}$ Ecole Nationale Supérieure des Mines de Saint Etienne, Centre de Microelectronique de Provence Georges \\ Charpak, 880 route de Mimet, 13120 Gardanne, Cedex, France
}

*corresponding author: tel:+33 (0)3 893375 15, Fax: +33 (0)3 89337505

e-mail: Gregory.Covarel@uha.fr

\section{Abstract:}

The mechanical properties of interfaces and more precisely the adhesion are of great importance to understand the reliability of thin film devices. Organic thin film transistors (OTFT) on flexible substrate are a new class of electronic components. Since these devices are flexible and intended for different fields of application like sensors and displays, they will undergo a lot of mechanical and thermal stress during their useful life. Moreover, interfaces play an important role in the electrical stability of these transistors. In this context, the adhesion of two organic submicronic thin films, semi conducting and dielectric respectively, deposited on polymeric substrate were investigated by scratch test method. This study demonstrates, the feasibility and selectivity of the scratch test as a tool for assessing the adhesion and the damage behaviour of ultra-thin organic film on flexible plastic substrate. The semi crystalline substrate presents a brittle cracking damage from a given strain, whereas when covered by the semi conducting thin film, the sample exhibits a more ductile behaviour. Moreover, this technique has proven to be sensitive enough to highlight the effects of a plasma treatment prior to deposition. 


\section{Introduction}

Interdisciplinary research efforts have led to the rapid development of Organic Thin Film Transistors (OTFTs) [1] with performances close to the one of a-Si TFTs [2]. OTFTs are made of submicronic organic and inorganic layers deposited on flexible substrate. Various materials $[3,4]$ and designs [5] have been investigated to improve the electrical functionality and stability of these devices. In a "top gate-bottom contacts" design, the semiconducting layer is deposited on the plastic substrate after the patterning of source and drain contacts. Then, the dielectric layer is laid on and, on top, a gate contact is done. These transistors show now good stability on shelf at ambient air [6]. Electrical and optical properties of these organic semiconductors have been extensively studied $[7,8]$, but their mechanical behaviour is not yet well understood. Since these transistors are flexible, they will undergo a lot of mechanical strains and stresses during their useful life. So the investigation of their reliability under mechanical stress is of great importance. Interfaces play a crucial role on the electrical functionality of devices and consequently the study of mechanical properties of interfaces is essential.

Many adhesion test techniques have been developed to measure adhesion energy at the interface, among which peel and pull-off tests are two widely used methods to test adhesion of thin films and coatings. The peel test is used in a variety of configurations, in which a thin strip is pulled away at some angle from the underlying substrate. Although the peel test offers simple test geometry for measuring adhesion strength $[9 ; 10]$, in the case of submicronic organic active layers of OTFT, the coating may tear due to the high stresses at the contact with the mechanical grips [11]. Moreover it is difficult to initiate the delamination with film thickness below $1 \mu \mathrm{m}$.

The pull-test allows a quantitative adhesion measurement, in which strain or energy can be extracted [12]. But it still suffers from several problems like the adhesive compatibility [13]. Indeed, pull-off test is performed by fixing, with an adhesive, a loading fixture to the surface 
of the film. Failure occurs along the weakest interface within the system which is often the adhesive/film interface because, in the present case, coatings have a poor adhesion to different types of adhesives.

Others specific adhesion test techniques have been developed like cross section indentation [14], four point bending [15], tensile loading [16] or blister adhesion test [17,18]. However, these methods require coating thickness of about several hundred of $\mu \mathrm{m}$, rigid substrate and difficult sample preparations, respectively.

Thus, a brief literature overview indicates adhesion measurements on OTFT are hard to implement. Indeed, in the submicron thickness range, analyses of coating damage is challenging considering relevant material properties such as toughness are unknown. Besides, numerous papers deal with adhesion properties determined by scratch test of various materials such as hard coatings or polymers [19-22]. However, few adhesion data are available for thin polymer coating deposited on polymeric substrate [23], and, none concerns submicronic electronic polymer coatings deposited on polymeric substrate so far. So, the present paper focuses on adhesion evaluation by scratch test which requires limited preparation to induce and quantify interface failure. Moreover the scratch test method is a useful tool because it is sensitive to several parameters (internal stresses, thickness, elastic properties...). The purpose of this study is to verify and investigate the feasibility and sensitivity of this method on two submicronic amorphous polymeric layers of OTFT deposited on semi crystalline substrate.

\section{Experimental}

In the present study, for simplification purpose, one type of specimen has been used. It consisted of a single thin layer deposited on a $125 \mu \mathrm{m}$ thick Teonex ${ }^{\circledR}$ Poly Ethylen Naphtalat (PEN) semicrystalline polymeric substrate. The substrate was obtained after a lamination process inducing anisotropic properties. Young Modulus were specified by 
DuPontTeijinFilms $^{\mathrm{TM}}$, at 5060 and $6240 \mathrm{MPa}$ for parallel and perpendicular directions to the laminating direction, respectively. Two thin films were considered. The first coating, namely $\mathrm{P} 1$ is a $200 \mathrm{~nm}$ thick triarylamine amorphous semiconducting polymer [24]. The second denoted as P2, is a $800 \mathrm{~nm}$ thick perfluoropolymer dielectric layer [25]. Films were deposited on PEN by spin coating, using a SCS 6800 spin coater apparatus, at room temperature at $3000 \mathrm{rpm}$ and $2000 \mathrm{rpm}$ for P1 and P2, respectively. The substrate size in the deposition process was $10 \times 10 \mathrm{~cm}^{2}$. Two deposition conditions were studied with and without Plasma Treatment (PT) prior to coating deposition. The plasma treatment, performed by means of a RIE Oxford Instrument Plasmalab apparatus, consists in a rapid reactive ion etch using $\mathrm{O}_{2}$ and $\mathrm{SF}_{6}$ gases, in order to improve the wettability of PEN surface [26]. Both layers present a glass transition temperature above $100^{\circ} \mathrm{C}$ preventing any change of structure during measurements. The layer thicknesses have been defined to optimize the electrical performances of the OTFT [27].

The adhesion properties were evaluated using a CSM Micro Scratch instrument scratch tester. The procedure was similar to that detailed elsewhere [19]. The scratch indenter was a diamond Rockwell C stylus with a spherical tip having a radius of $200 \mu \mathrm{m}$. The indenter was chosen in order to operate in the better accuracy range of experimental apparatus in terms either of load or sensor sensibility. For the $5 \mathrm{~mm}$ scratch length, the applied load was progressively increased from $0 \mathrm{~N}$ to $10 \mathrm{~N}$ at a rate of $50 \mathrm{~N} / \mathrm{min}$. Such an intermediate scratch speed was used to be sensitive to elastic, plastic and fracture contributions, because polymer materials are sensitive to time dependant effects and as reported by Barletta et al. plasticity tends to decrease and fracture contribution to increase when scratch speed is increased [28]. Five measurements were performed at room temperature for each sample and an average value of the critical load is obtained. After the test, a critical load (Lc) where failure occurred in a particular mode was determined by post-mortem observation of the scratch track using an optical microscope. The error on the Lc determination is due to two main contributions. A 
major contribution is due to statistical errors and on the other hand the position accuracy of the sample under the microscope is $1 \mu \mathrm{m}$ which results in an error of some $\mathrm{mN}$. Since statistical errors represent the major contribution, scattering given below corresponds to the standard deviation. In addition, Acoustic Emission signals were recorded during the test by a sensor attached to the load arm.

Scanning Electron Microscopy (SEM) was performed using a CARL ZEISS-Ultra 55 apparatus and elemental analyses were carried out by Energy-Dispersive X ray (EDX) using an OXFORD INCA system.

\section{Results and discussion}

Taking into account the load range, the tip geometry, material properties and layer thicknesses, the mean penetration depth at $10 \mathrm{~N}$ is of about $40 \mu \mathrm{m}$ for all specimens studied in the following. In addition, due to viscoplastic nature of materials a pile up is formed ahead and on the edges of the track.

The damage sequences of specimen PEN, P1 layer on PEN and P2 layer on PEN are shown in Fig. 1. For all materials tested, there is only a small amount of deformation observed under low load and stress level. This includes fully recoverable elastic deformation, time dependant viscoelastic deformation, and a small amount of non-recoverable plastic deformation resulting from compressive indentation, tentatively termed "mar" [29].

Fig.1:

The damage sequence observed for the PEN substrate, at room temperature, is presented in

Fig. 1-a. At an early stage of the scratch, only mar deformation is observed up to about $1.7 \mathrm{~N}$. 
Progressively forward semi circular features occurred in the scratch track as seen at $4 \mathrm{~N}$. Actually, plastic deformation in front of the scratch tool produced a pile up of polymer which offers a resistance to the advancing indenter. Such material is submitted to compressive stresses, at the same time, material under the indenter is taken in a strong tensile state [30,31]. Hence, the observed damage is due to the compressive stresses ahead of indenter and the plastic flow around it as indicated by the parabolic morphology [32,33]. At higher load, microcracking occurs in the track transversally to the scratch direction, as evidenced at $5 \mathrm{~N}$ and more visible at $7 \mathrm{~N}$, indicating a brittle behaviour [34]. That is very likely due to the semi crystalline nature of this polymer [35]. The mean load of crack appearance is $4.3 \pm 0.3 \mathrm{~N}$. The failure events of P1 layer deposited on PEN substrate, shown in Fig. 1-b, present some differences. At low load, only mar deformation is observed up to about 1.8 N. For higher loads (see for instance $4 \mathrm{~N}$ ), the forward semi-circular feature is present, but with increasing load, a stick-slip phenomenon is clearly visible in the bottom of the scratch track. A stick slip phenomenon occurs when the indenter experiences change during the tip movement. Thus, formation and breakage adhesion between tip and coating occurs repeatedly. More over it becomes more significant when normal load increases $[29,36]$. In the present case, the strain energy release process appears by localized ductile tearing of superficial coating due to the interaction between tangential load and the viscoelastic nature of polymer; characterizing the ductile behaviour of the system [36]. Nevertheless, it is worth noting that forward semicircular feature concerns the coating and the substrate too, since the layer remains adhering to the substrate even for higher loads studied. In addition, no evidence of cutting mark or crack could be found for this specimen and the mean critical load, defined as the first appearance of the stick-slip phenomenon, is $5.1 \pm 0.5 \mathrm{~N}$. This behaviour is confirmed by a SEM observation presented in fig 2-a, evidencing the periodic tearing of superficial coating in the bottom of the scratch track. Although only semi conducting coating contains Nitrogen and 
not the substrate, an EDX analysis was not relevant considering the resolution of the apparatus since $\mathrm{N} \mathrm{K \alpha} \square$ energy is very close to the $\mathrm{C}$ one.

Fig2:

Scratch results for the P2 coating deposited on PEN substrate are reported in Fig. 1-c. The beginning of the damage sequence is the same than for the two previous specimens i.e. mar and subsequently parabolic deformation, but for increasing load a sudden and large delamination of the superficial coating is observed. This delamination spread widely outside the scratch track. For this sample, the critical load, defined as the lowest load inducing the delamination, is $4.2 \pm 0.3 \mathrm{~N}$.

In the area of critical load a SEM analysis has been performed, a picture taken at an higher magnification shown in figure 2-b bore out optical observations describing a sudden damage of the coating. More over a F K $\alpha$ cartography presented in figure 2-c indicates clearly that the perfluoropolymer coating is no more detectable in the scratch track after the critical load. The shear stresses in the scratch subsurface region may be responsible for the delamination of the superficial coating [36].

It has to be noticed that we have checked on other specimens, not shown here, that the slight discrepancy of Young Modulus measured on PEN substrate for both directions, i.e. parallel and perpendicular to the laminating direction, did not induce any variation neither in the damage mode nor in critical load for studied coatings.

Fig. 3: 
In a second set of experiments, the effect of a plasma treatment (PT) on substrate prior to coating deposition was investigated. The damage sequence of the P1 coating deposited on PEN substrate with PT is shown in Fig. 3-a. It is quite identical to the one obtained without PT (Fig. 1-b). Indeed, at low load only the groove of the indenter is observed up to about 1.5 N. The forward parabolic features are identified progressively and as increasing load, a stickslip phenomenon is observed at the bottom of the scratch track. The first apparition of a stickslip phenomenon is detected for a critical load of about $4.2 \pm 0.5 \mathrm{~N}$; a slightly lower critical load, than for the coating without PT, indicating the weak influence of pretreatment. Nevertheless, no brittle fracture was detected.

The failure sequence of P2 coating deposited on PEN substrate with PT is presented in Fig. 3-b. Contrarily to the case of the P1 coating, the damage sequence with and without PT is different. Actually, with PT, the mar deformation is still observed up to about $1.5 \mathrm{~N}$, followed by the forward parabolic feature. But as the load increases, the total delamination of the superficial coating is no more present. Only few localized and cohesive damages appear in the bottom of the scratch track as seen for instance at $6 \mathrm{~N}$ or $7 \mathrm{~N}$. The lowest load at which it occurs is about $5.6 \pm 0.2 \mathrm{~N}$. The coating remains adhering to the substrate on the edge of the scratch track even at $10 \mathrm{~N}$. For the P2 coating, the plasma treatment improves the adhesion of the thin film.

Fig. 4:

Acoustic Emissions (AE) recorded during tests are plotted for each specimen in Fig. 4. At low load, no AE signal is detected for all specimens. Then, the AE evolution depends on the failure mechanism. For PEN, the AE increases concomitantly with the apparition of cracks 
transversally to the scratch tracks confirming the brittle behaviour of this failure mode. For both $\mathrm{P} 1$ coatings, with and without PT, no evolution of the AE is detected all along the test, indicating a more ductile damage. Concerning the P2 coating deposited on PEN substrate, the AE increases simultaneously with the delamination observed by optical microscopy. But when the substrate is plasma treated prior to deposition the signal remains flat, evidencing the enhancement of adhesion properties.

\section{Conclusion}

In the present paper, we have reported results concerning submicronic organic coatings deposited on a polymeric substrate. We stated that covering a semicrystalline polymer, which exhibits brittle behaviour, by a submicronic amorphous polymer could change drastically the damage behaviour. The scratch test method is a semi quantitative, sensitive and reproducible tool to characterize the adhesion of polymer coating on a polymer substrate even for thin layers of a few hundred of nanometres. The scratch test was capable of highlighting clear differences between the mechanical behaviour of uncoated and coated substrates. But it also means that direct comparison between the critical load values of different systems is not possible: each critical load is indeed related to different failure mechanism. For direct quantification purpose, it is necessary to induce a unique failure mechanism, the use of a sharper tip would be therefore useful, because when the tip radius is smaller, the maximum stress is located closer to the surface.

\section{Acknowledgement:}

CEA LITEN is gratefully acknowledged for providing specimens. Ms Brosse is acknowledged for technical support. 


\section{References:}

[1] H. Sirringhaus, Adv. Mat. 17 (2005) 2411-2425.

[2] J. E. Anthony, M. Heeney, B.S. Ong, MRS Bull. 33 (2008) 698-705.

[3] N. Karl, Synthetic Metals 133 (2003) 649-657.

[4] H. Inokuchi, Organic Electronics 7 (2006) 62-76.

[5] D. Gupta, M. Katiyar, D. Gupta, Organic Electronics 10 (2009) 775-784.

[6] J.M. Verilhac, M. Benwadih, A.L. Seiler, S. Jacob, Organic Electronics 11 (2010) 456562.

[7] H. Sirringhaus, Adv. Mater. 21 (2009) 3859-3873.

[8] L. Léontie,R. Danac, Scripta Mater. 54 (2006) 175-179.

[9] A. N. Gent, G. R. Hamed, J. of Appl. Polym Sci. 21 (1977) 2817-2831.

[10] M. D. Thouless, H. M. Jensen, J. of Adhesion 38 (1992) 185-197.

[11] Y. H. Lai, D. A. Dillard, J. of Adhesion 56 (1996) 59-78.

[12] C. T. Sun, W. Qian, Int. J. Solids Struct. 34 (1997) 2595-2609.

[13] H. Yu, J.W. Hutchinson, Thin Solid Films 423 (2003) 54-63.

[14] J. Lesage, P. Demarecaux, O. Bartier, G. Mesmacque, Revue de Métallurgie, 90, 12 (1993) 1655-1663.

[15] R. H. Dauskardt, M. Lanea, Q. Mab and N. Krishnac, Engineering Fracture Mechanics 61 (1998) 141-162.

[16] H-J. Kim, M-W. Moon, D-I. Kim,K.-R Lee, K.-H. Oh, Scripta Mater. 57 (2007) 10161019.

[17] H.M. Jensen, M.D. Thouless, Int. J. Solids Struct. 30 (1993) 779-795.

[18] Y. H. Lai, D. A. Dillard, J. Adhesion Sci. Technol. 8 (1994) 663-678.

[19] L. Marot, G. Covarel, M. H. Tuilier, R. Steiner, P. Oelhafen, Thin Solid Films, 516 (2008) 7604-7608.

[20] R. L. Browning, G.-T. Lim, A. Moyse, H.-J. Sue, H. Chen, J. D. Earls, Surf. Coat. Technol. 201 (2006) 2970-2976.

[21] C. Gauthier, A.-L. Durier, C. fond, R. Schirrer, Trib Int. 39 (2006) 88-98.

[22] X. Zhang, L. Hu, D. Sun, Acta Mater 54 (2006) 5469-5475. 
[23] I. Demirci, C. Gauthier, R. Schirrer Thin Solid Films 479 (2005) 207-215.

[24] E. M. Barea, G. Garcia-Belmonte, M. Sommer, S. Hüttner, H.J. Bolink, M. Thelakkat, Thin Solid Films 518 (2010) 3351-3354.

[25] T. Umeda, D. Kumaki, S. Tokito, Organic Electronics 9 (2008) 545-549.

[26] D. Hegemann, H. Brunner, C. Oehr, Nucl. Instr. and Meth. in Phys. Res. B 208 (2003) 281-286.

[27] D. Boudinet, M. Benwadih, S. Altazin, R. Gwoziecki, J.M. Verilhac, R. Coppard, Organic Electronics 11 (2010) 291-298.

[28] M. Barletta, A. Gisario, L. Lusvarghhi, G. Boleli, G. Rubino, Appli. Surf. Sci. 254 (2008) 7198-7214.

[29] H. Jiang, R. Browning, H-J. Sue, polymer 50 (2009) 4056-4065.

[30] M. Wong, G.T. Lim, A. Moyse, J. N. Reddy, H.-J. Sue, Wear 256 (2004)1214-1227.

[31] J. S. S. Wong, H.-J. Sue, K.-Y. Zeng, R. K. Y. Li, Y. W. Mai, Acta Mater. 52 (2004) 431-443.

[32] M. Barletta, A. Gisario, G. Rubino, L. Lusvarghi, Progress in organic coatings 64 (2009) 247-258.

[33] A. Dasari, J. Rohrmann, R. D. K. Misra, Mater. Sci. Eng. A354 (2003) 67-81.

[34] C.-W. Yang, J. -W. Park, Surf. Coat. Technol. 204 (2010) 2751-2766.

[35] W. A. MacDonald, M.K. Looney, D. MacKerron, R. Eveson, R. Adam, Journal of the Society for Information Display 15 (2007) 1075-1083.

[36] A. Dasari, Z.-Z. Yu, Y. W. Mai, Acta Mater. 55 (2007) 635-646. 
Figure Captions :

Fig. 1 : Main part of the scratch track on PEN (a), P1/PEN (b) and P2/PEN (c) observed by optical microscopy.

Fig. 2: SEM micrographs of P1 (a) and P2 (b) on PEN in the scratch track and corresponding F K $\alpha \square$ EDX cartography of P2 on PEN (c). Arrows indicate the scratch direction.

Fig. 3 : Main part of the scratch track on P1 (a) and P2 (b) deposited on Plasma Treated PEN observed by optical microscopy.

Fig. 4 : Acoustic Emission recorded during a scratch measurement of PEN, P1/PEN, P2/PEN and P1/Plasma Treated PEN and P2/ Plasma Treated PEN. For visualisation, curves were arbitrary shifted. 


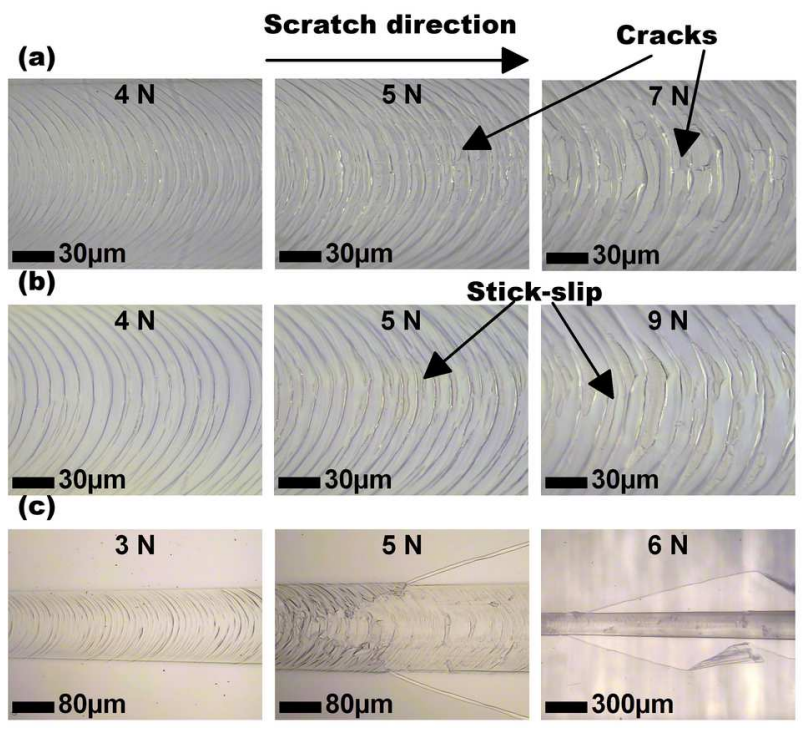

Fig 1 

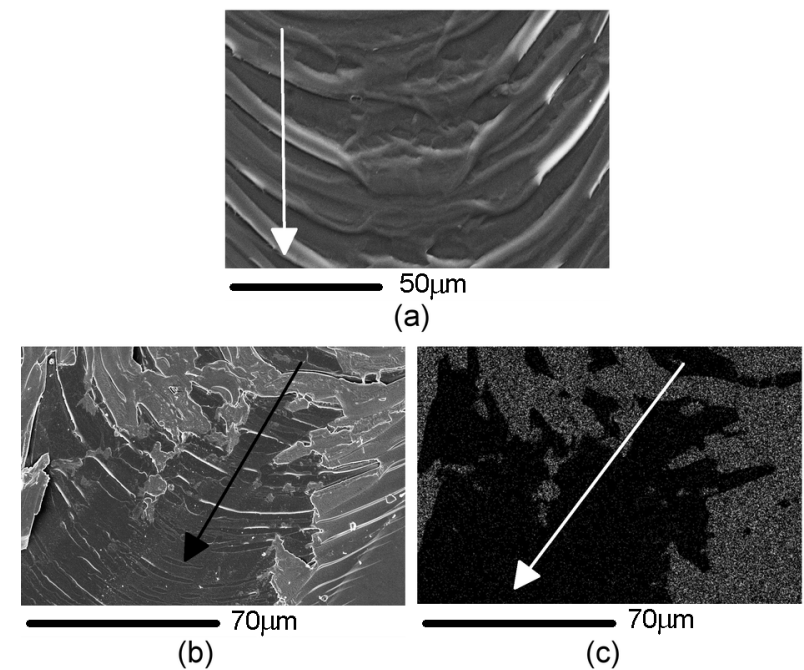

Figure 2. 


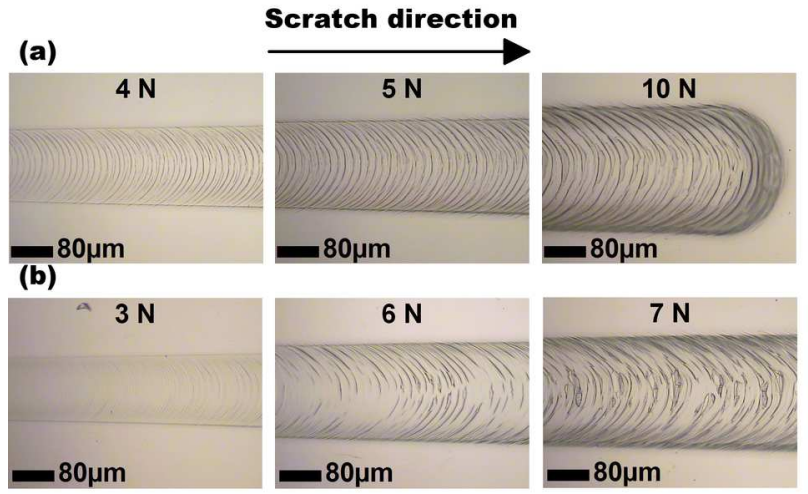

Figure 3. 


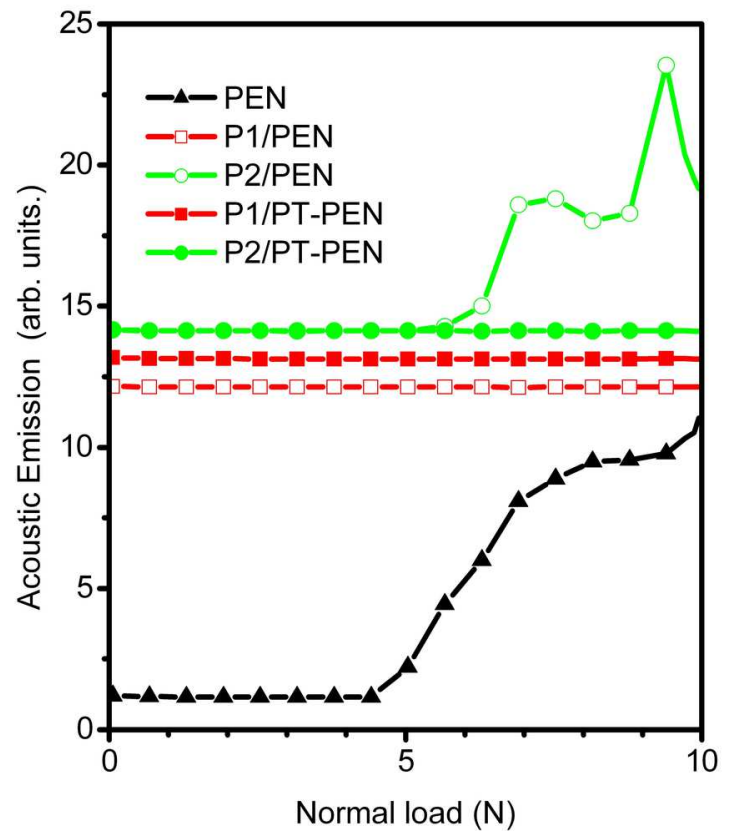

Figure 4. 